\title{
Evolución y Características de la Demanda Turística en la Reserva Natural de Punta Tombo, Chubut, Argentina
}

\author{
Evelution and Characteristics of Teurism Demand in the \\ Natural Protected Area of Punta Tombo, Chubut, Argentina
}

\section{Piedad María Losano}

\begin{abstract}
RESUMEN: El presente trabajo se centra en el análisis de la demanda turística del Área Protegida Punta Tombo, ubicada a 107 kilómetros al sur de la ciudad de Trelew, Argentina. Éste se efectuó a partir de datos obtenidos de la venta de entradas para el ingreso a la misma y comprende el período 1991/92 a 2000/ 01 . Las principales conclusiones obtenidas fueron: tendencia creciente de la demanda a largo plazo; la demanda por parte de turistas extranjeros constituye el $40 \%$; esta se concentra principalmente en los meses de noviembre y diciembre; las visitas tienen una estacionalidad muy marcada, donde destacan los meses de octubre y enero; los cruceros turísticos constituyen un importante componente de la demanda. Aproximadamente el $40 \%$ de sus pasajeros se desplaza hasta el AP.
\end{abstract}

PALABRAS CLAVE: demanda turística; evolução e características; reserva natural; Punta Tombo; Chubut; Argentina.

ABSTRACT: This work is centered in the tourism demand to the Protected
Area Punta Tombo, situated aproximatwly $107 \mathrm{~km}$ South of Trelew, Argenti-
na. It was done with the information obtained from the sale of entrance
tickets from 1991/92 to 2000/01. We obtained the following main conclusions:

1. Licenciada en Economía. Funcionaria de la Secretaría de Turismo de la Provincia del Chubut. Cargo actual:Directora de Control de Calidad y Servicios. Docente e Investigadora de la Universidad Nacional de la Patagonia San Juan Bosco. Miembro activo del GETACE (Instituto de Investigación en Temas Ambientales en Ciencias Económicas). Consultora en temas económicos-turísticos. Contacto: Pellegrini 1509 - 9100 - Trelew, Chubut - R. Argentina; e-mail: plosano@hotmail.com. 
a growing tendency of Tourism for a large period of time ahead; foreign Tourism goes up to $40 \%$ of the totality; it is mainly concentrated in the months of November and December; visits have a defined tendency: October and January are the outstanding months; tourism cruises constitute an important part of tourists affluence. Approximately $40 \%$ of the passengers visit this Protected Area.

KEYWORDS: tourism demand; evolution and characteristics; natural protected area; Punta Tombo; Chubut; Argentina.

\section{Introducción}

El presente trabajo forma parte del Proyecto de Investigación Gestión Ambiental de Áreas Protegidas. Desarrollo de un Modelo de Gerenciamiento Ambiental para la Reserva Natural Punta Tombo, Chubut, Argentina, que se está llevando a cabo en el ámbito de la Facultad de Ciencias Económicas de la Universidad Nacional de la Patagonia San Juan Bosco.

El objetivo es desarrollar un modelo de gestión ambiental que sea aplicable a la actividad turística que se desenvuelve dentro de las Áreas Naturales Protegidas (en especial, la Reserva Natural Turística de Punta Tombo), el que debe contemplar las particularidades ambientales del sitio, potenciando su conservación e incrementando el nivel de satisfacción del turista. A tal fin, se busca proponer un modelo de gerenciamiento ambiental que integre el planeamiento, gestión y control ambiental, incluyendo:

- la formulación de objetivos, políticas y estrategias de prevención y minimización;

- el diseño de programas de gestión ambiental tendientes a lograr una mejora continua y una disminución de los riesgos potenciales;

- el establecimiento de programas de control ambiental que permitan asegurar el cumplimiento de las responsabilidades ambientales y de la legislación vigente.

El objetivo del presente trabajo, en particular, es analizar la demanda turística al Área Natural Protegida de Punta Tombo, especialmente en lo que se refiere al crecimiento histórico y al comportamiento de algunas variables clave de esta demanda (composición, ciclos anuales, ingresos directos, carga diaria de la Reserva).

\section{Área de Estudio}

La Reserva Natural Turística de Objetivo Específico Punta Tombo está ubicada a 107 kilómetros al sur de la ciudad de Trelew (Provincia del Chubut). Es el refugio de una de las más importantes colonias de avifauna marina del país y la mayor colonia continental de pingüinos de Magallanes que, en plena temporada, supera los 700 mil ejemplares. La RNPT es una estrecha y pedregosa franja que abarca unas 210 hesctáreas y que penetra en el mar unos 3,5 kilómetros. (Provincia del Chubut, 1980).

Esta Reserva fue creada originalmente como Reserva Biológica en el año 1972 mediante Decreto Provincial n 2870/72. Posteriormente, en el año 1983, por Ley no 2161, se crea el Sistema Provincial de Conservación del Patrimonio Turístico de la Provincia del Chubut y se incorpora Punta Tombo como una Reserva Natural Turística de Objetivo Específico, para proteger a los pingüinos de Magallanes y a las especies que con ellos conviven.

El pingüino de Magallanes es una especie migratoria. A partir de la segunda quincena de septiembre, y hasta el mes de marzo de cada año, la colonia desarrolla una total actividad. Luego, iniciando su período de vida pelágica, abandona la costa del Chubut hasta la siguiente primavera. Durante su período de estadía en la colonia, se pueden distinguir varias etapas, cada una de ellas genera su propia atracción y responde a los distintos grados de evolución del ciclo biológico vital del pingüino:

- llegada y selección del nido;

- demarcación de territorios y formación de parejas;

- posturas de huevos y su defensa de las aves predadoras;

- llegada de los juveniles, nacimiento de pichones y alimentación;

- cambio de plumas y preparación para la partida.

\section{Metodología}

El estudio que posibilitó el análisis que se presenta se efectuó sobre la base de recopilación de datos que surgen del control contable-administrativo de las ventas de entradas para el ingreso a la Reserva Natural, existente en la Secretaría de Turismo y Áreas Protegidas de la Provincia del Chubut.

Las ventas de los billetes, durante el período de análisis, se realizaron en la misma Reserva, bajo responsabilidad del guarda fauna a cargo de la misma, y en la Dirección de Turismo de la ciudad de Puerto Madryn. Para su efectivo control, se lleva a cabo un registro minucioso de las ventas diarias según nú- 
mero de billetes y valor de los mismos (como se verá más adelante, el criterio para la diferenciación en el valor de las entradas fluctuó a lo largo del tiempo, influyendo en la potencialidad del análisis de la demanda a partir de estos factores).

La recopilación de estos datos y su formalización sistemática para el análisis corrieron por cuenta de la autora del presente trabajo y son el resultado de varios años de consecución en esta tarea, logrando, así, obtener una amplia base de registro histórico de los ingresos de turistas y residentes a la RNPT.

\section{Resultados}

Si bien existen algunos registros de ingreso de visitantes a la Reserva a partir del año 1979, estos son aislados; por lo tanto, esta discontinuidad de la serie condicionó el análisis al período comprendido entre las temporadas 1991/92 y 2000/01.

\section{Expansión de la demanda}

La Provincia del Chubut se proyecta turisticamente a partir de la difusión de los atractivos naturales constituidos por asentamientos de importantes colonias de aves y mamíferos marinos sobre su costa atlántica. Para ellos, inicia, en los últimos años de la década de los '60, un ambicioso programa de conservación y protección, creando un sistema de Reservas Naturales bajo administración provincial.

A lo largo de estos años, quizás como consecuencia de la rápida evolución del turismo de naturaleza en todo el mundo y la avidez dela demanda por descubrir nuevas motivaciones para su desplazamiento, se observó un interesante fenómeno de intercambio y traslape en el gusto de los turistas hacia las diferentes especies que pueblan estas costas.

Durante esos primeros años (década de los '70) el principal atractivo turístico fue el elefante marino del sur, cuyo hábitat está localizado en la Reserva de Punta Norte (Península Valdés); luego, en la década siguiente, la especie que se puso en valor turístico fue el pingüino de Magallanes, en la Reserva Natural Turística de Punta Tombo.

Posteriormente, ya en los años '90, la ballena franca austral comienza a posicionarse como un atractivo turístico de características internacionales $y$, rápidamente, alcanza la jerarquía de los dos anteriores (Pascotto, Tagliorette y Losano, 1990).
Estas tres especies siguen siendo los atractivos convocantes de las corrientes turísticas, mientras que los lobos marinos y las aves costeras son atractivos complementarios. En los últimos años, la observación de orcasy de toninas overas comenzó a formar parte de los itinerarios.

Los primeros datos cuantitativos de visitantes a la Reserva Natural Turística de Punta Tombo se remontan al año 1979, con un total de 4.993 individuos. Esta demanda evolucionó rápidamente en los años subsiguientes, llegando a triplicar esa cifra inicial ya en 1981 (Losano, 1986).

Para la temporada 1991/92, el número de visitantes superó la cifra de 29 mil personas. Este comportamiento ascendente de la demanda continuó (con algunos altibajos) hasta la temporada 1997/98, en la que superó los 75 mil visitantes (máximo histórico de la Reserva). A partir de esa fecha, se inicia un proceso de declinación, en lo que se refiere a cantidad de visitantes (Tabla 1 y Figura 1).

En la Tabla 1, se verifican los ingresos de visitantes ordenados según la temporada turística, que se extiende - coincidentemente con la presencia de pingüinos en el área-, entre los meses de septiembre de un año a marzo/abril del año siguiente. Estos datos comprenden el total de ticket vendidos más un porcentaje (estimado en un $15 \%$ ) en concepto de menores de 12 años y otros visitantes exentos de pago (periodistas, invitados, funcionarios). La excepción está dada por la última temporada, debido a que la exención para niños se redujo a los menores de 6 años. En consecuencia, el porcentaje estimado disminuyó a un 7\%.

En la Figura 1, se observan los totales de temporada, discriminando los dos conceptos mencionados en el párrafo precedente.

Si bien la tendencia indica un crecimiento a largo plazo en el número de visitantes, el mismo ha tenido un comportamiento bastante fluctuante, experimentando en algunos años crecimientos muy significativos (hasta del $48 \%$ ) y en otros estancamiento y, aún, declinación. Se observa la presencia de dos ciclos: el primero, creciente, se extiende desde su inicio hasta la temporada 1997/ 98 y el segundo, decreciente, desde 1998/99 hasta la última temporada.

La línea de regresión que representa el comportamiento promedio de la serie completa responde a la siguiente ecuación:

$$
y=37819+3164,1 x
$$

con un

$$
r^{2}=0,456
$$




\begin{tabular}{|c|c|c|c|c|c|c|c|c|c|c|}
\hline 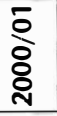 & $\begin{array}{l}a े \\
\stackrel{y}{y}\end{array}$ & $\overline{\mathfrak{N}}$ & $\begin{array}{l}\bar{n} \\
\sigma\end{array}$ & $\begin{array}{l}\hat{\tilde{n}} \\
\tilde{\sigma}\end{array}$ & 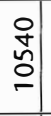 & $\begin{array}{l}a \\
\hat{n} \\
0\end{array}$ & $\begin{array}{l}m \\
\stackrel{d}{\sim}\end{array}$ & $\underset{\infty}{\mathbb{N}}$ & $\begin{array}{l}\infty \\
\tilde{n} \\
0 \\
\bar{n} \\
\end{array}$ & $\bar{i}$ \\
\hline ষ্ণ & $\begin{array}{l}\hat{\sigma} \\
\text { m. } \\
\hat{b}\end{array}$ & $\begin{array}{l}\hat{\sigma} \\
\dot{\square}\end{array}$ & 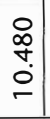 & $\begin{array}{l}m \\
\bar{\sigma} \\
\dot{r}\end{array}$ & $\begin{array}{l}\infty \\
\infty \\
\square \\
\square\end{array}$ & $\begin{array}{c}\frac{m}{\nu} \\
\sigma\end{array}$ & $\begin{array}{l}\tilde{y} \\
\stackrel{⿲}{\mathbf{v}}\end{array}$ & $\begin{array}{l}\Delta \\
0 \\
-\end{array}$ & 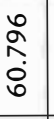 & $\begin{array}{l}0 \\
\infty \\
\\
\end{array}$ \\
\hline $\begin{array}{l}\text { ă } \\
\text { aे } \\
\alpha\end{array}$ & 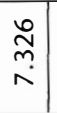 & $\begin{array}{l}\tilde{J} \\
\stackrel{\Gamma}{ }\end{array}$ & $\begin{array}{l}\infty \\
\infty \\
\infty \\
\dot{\square}\end{array}$ & $\begin{array}{l}\infty \\
m \\
n \\
0\end{array}$ & $\begin{array}{l}\infty \\
0 \\
0 \\
\dot{n} \\
\end{array}$ & $\begin{array}{c}\tilde{N} \\
\stackrel{2}{\infty} \\
\infty\end{array}$ & $\begin{array}{l}\bar{n} \\
m\end{array}$ & \begin{tabular}{r}
\multirow{y}{f}{} \\
-
\end{tabular} & 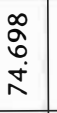 & $\begin{array}{l}\infty \\
\vdots \\
i \\
i\end{array}$ \\
\hline 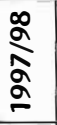 & $\begin{array}{l}\hat{y} \\
0 \\
\dot{0}\end{array}$ & $\begin{array}{l}\tilde{N} \\
\stackrel{\dot{J}}{\leftarrow}\end{array}$ & $\begin{array}{l}\infty \\
0 \\
\dot{+} \\
-\end{array}$ & $\begin{array}{l}\infty \\
\stackrel{u}{u} \\
b\end{array}$ & $\begin{array}{l}\hat{\mathfrak{v}} \\
\stackrel{+}{\sim}\end{array}$ & $\begin{array}{l}\stackrel{o}{+} \\
\dot{=}\end{array}$ & $\begin{array}{l}\underset{+}{\sim} \\
\stackrel{+}{+}\end{array}$ & \begin{tabular}{l}
$\tilde{N}$ \\
\multirow{r}{-}{} \\
-
\end{tabular} & 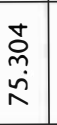 & $\hat{\bar{m}}$ \\
\hline ๙ু & 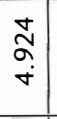 & $\begin{array}{l}m \\
\& \\
\dot{0} \\
-\end{array}$ & $\begin{array}{c}\stackrel{0}{0} \\
m \\
\stackrel{0}{-}\end{array}$ & $\begin{array}{l}m \\
\infty \\
\dot{+} \\
\dot{n}\end{array}$ & $\begin{array}{l}\bar{m} \\
\stackrel{\underline{v}}{ }\end{array}$ & $\begin{array}{l}0 \\
0 \\
0 \\
0 \\
0\end{array}$ & $\begin{array}{l}\tilde{\tilde{o}} \\
\stackrel{+}{\dot{*}}\end{array}$ & & $\begin{array}{l}\text { Vै } \\
\frac{1}{\pi} \\
\text { in }\end{array}$ & $\bar{\nabla}$ \\
\hline 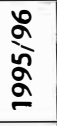 & $\begin{array}{l}\underset{y}{W} \\
\infty \\
\dot{m}\end{array}$ & $\begin{array}{c}\bar{\sigma} \\
\infty \\
\sigma\end{array}$ & $\begin{array}{l}\hat{\tilde{N}} \\
\hat{a}\end{array}$ & $\begin{array}{l}\hat{a} \\
\infty \\
\dot{m}\end{array}$ & $\begin{array}{l}\tilde{O} \\
0 \\
\dot{I} \\
\simeq\end{array}$ & 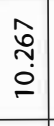 & 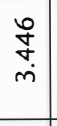 & $\stackrel{n}{\text { mे }}$ & 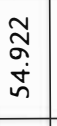 & $\stackrel{+}{-}$ \\
\hline $\begin{array}{l}\text { ๙̊ } \\
\text { ă } \\
\text { ă }\end{array}$ & $\begin{array}{l}\infty \\
\dot{\sigma} \\
\dot{+}\end{array}$ & $\begin{array}{l}\hat{n} \\
m \\
\check{\sigma} \\
=\end{array}$ & $\begin{array}{l}\bar{m} \\
\tilde{\sigma} \\
\sigma\end{array}$ & $\begin{array}{l}\mathscr{0} \\
0 \\
0 \\
\dot{m}\end{array}$ & 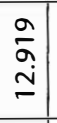 & \begin{tabular}{l}
$\hat{\jmath}$ \\
\multirow{\sigma}{}{} \\
$\sigma$
\end{tabular} & $\begin{array}{l}\frac{O}{\square} \\
\dot{m}\end{array}$ & 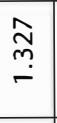 & 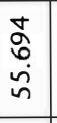 & $\stackrel{0}{=}$ \\
\hline 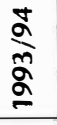 & $\begin{array}{l}\tilde{\partial} \\
\dot{m}\end{array}$ & $\begin{array}{l}\hat{m} \\
\text { ñ } \\
\stackrel{0}{-}\end{array}$ & $\begin{array}{c}\bar{\lambda} \\
\infty\end{array}$ & $\begin{array}{l}\infty \\
\stackrel{+}{+} \\
\dot{+}\end{array}$ & $\begin{array}{l}\stackrel{\sim}{\infty} \\
\stackrel{n}{n} \\
\end{array}$ & $\begin{array}{l}N \\
\infty \\
\sigma \\
\sigma\end{array}$ & 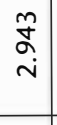 & $\frac{\bar{\sigma}}{\bar{i}}$ & $\begin{array}{l}\frac{\tilde{D}}{n} \\
\text { n் }\end{array}$ & $\stackrel{a}{\hat{\sigma}}$ \\
\hline $\begin{array}{l}\text { à } \\
\text { ลे } \\
\text { a }\end{array}$ & ñ & $\begin{array}{l}\infty \\
0 \\
\stackrel{\sim}{+} \\
+\end{array}$ & $\begin{array}{l}0 \\
\tilde{n} \\
\\
n\end{array}$ & $\begin{array}{l}\tilde{Z} \\
\dot{+}\end{array}$ & $\begin{array}{l}\sigma \\
\text { o. } \\
\sigma\end{array}$ & $\begin{array}{l}\hat{n} \\
\hat{\sigma}\end{array}$ & $\begin{array}{l}m \\
\stackrel{0}{ } \\
\stackrel{v}{1}\end{array}$ & & $\begin{array}{l}\underset{N}{N} \\
\stackrel{n}{m}\end{array}$ & ָั \\
\hline$\frac{\alpha}{\alpha}$ & â & \begin{tabular}{l}
$\stackrel{2}{\nwarrow}$ \\
\multirow{+}{*}{}
\end{tabular} & 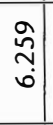 & $\begin{array}{l}\bar{\infty} \\
\hat{i} \\
\mathbf{v}\end{array}$ & $\begin{array}{l}n \\
\hat{\sigma} \\
\dot{b}\end{array}$ & $\begin{array}{l}0 \\
\infty \\
\\
\dot{n}\end{array}$ & 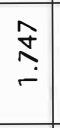 & & $\begin{array}{l}\text { जे } \\
\text { సे }\end{array}$ & \\
\hline$\breve{\Sigma}$ & 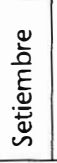 & 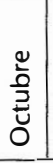 & 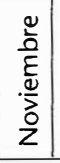 & 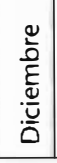 & 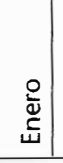 & 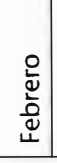 & 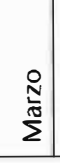 & $\begin{array}{l}\overline{\mathbf{E}} \\
\overline{\mathrm{Z}}\end{array}$ & $\begin{array}{l}\overrightarrow{5} \\
\stackrel{5}{L}\end{array}$ & 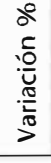 \\
\hline
\end{tabular}

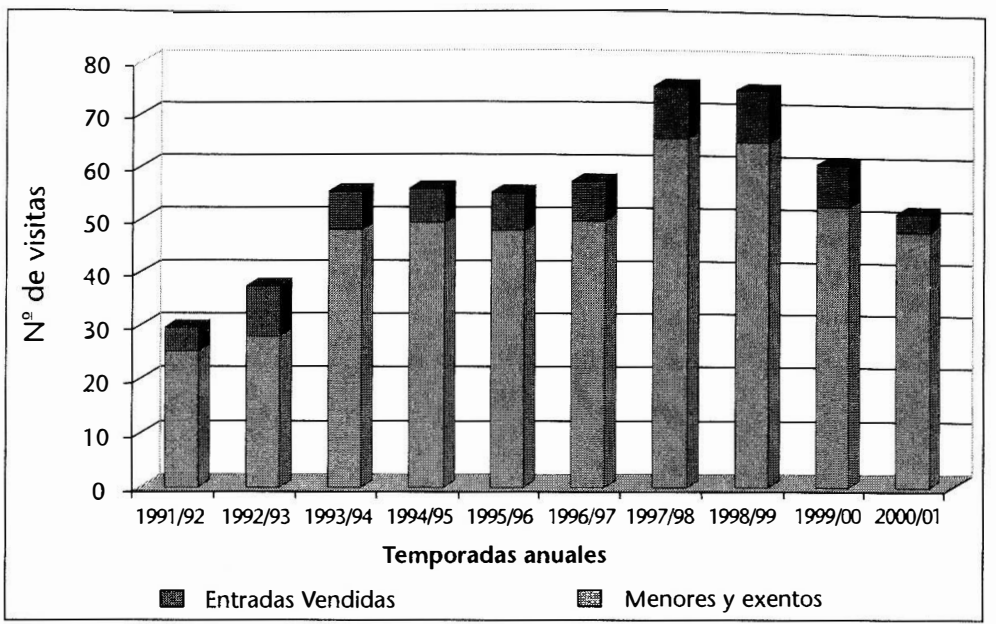

Figura 1 Total de visitantes a la RNT Punta Tombo (Temporadas 1991/92 - 2000/02)

En que las correspondientes variables significan:

- y: cantidad estimada de turistas;

- $x:$ año;

- 3164,1: parámetro que representa el incremento anual promedio en el número de turistas;

- $\mathrm{r}^{2}$ : coeficiente de determinación que da un valor intermedio, lo cual indica la existencia de una importante dispersión entre el valor observado y el valor estimado de la variable turista ${ }^{2}$.

Pese a la declinación observada en el número de turistas durante las tres últimas temporadas, la tendencia sigue demostrando un comportamiento ascendente (Figura 2).

2. $\mathrm{r}^{2}$ indica en qué proporción la variable regresada está vinculada a la variable regresora $(\mathrm{x})$. Su valor oscila entre 0 y 1 ; más cerca esté de 1 , mejor es el ajuste. 


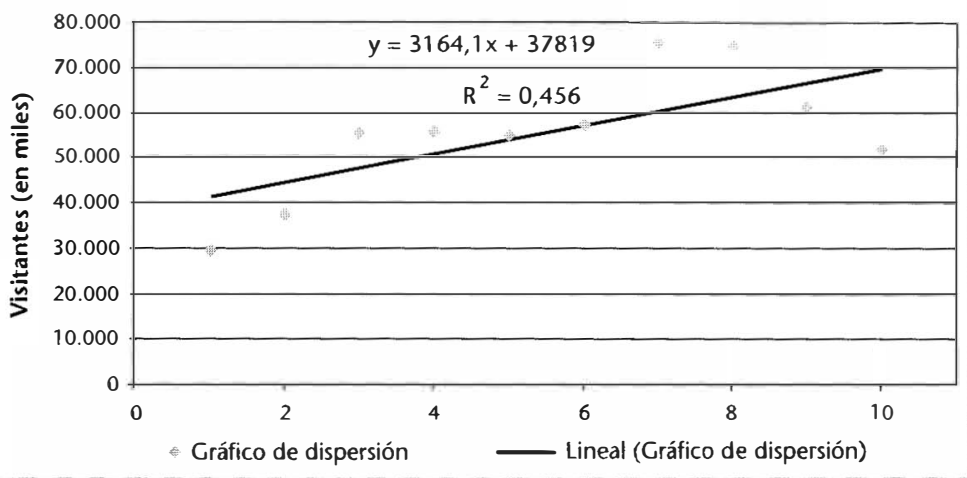

Figura 2 Visitantes a la RNT Punta Tombo (diagrama de dispersión y ajuste lineal)

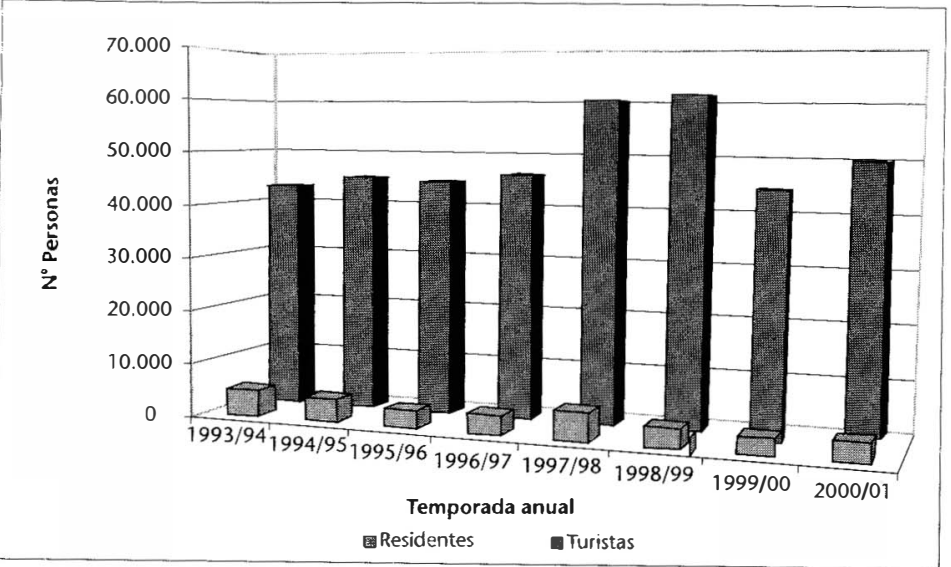

Figura 3 RNT Punta Tombo - Distribución entre turistas y residentes

\section{Origen de los visitantes}

A partir de la temporada 1993/94, el sistema de cobro del ingreso permitió diferenciar los visitantes entre residentes de la Provincia y turistas. En la Figura 3, se muestra la composición de ambos subgrupos. Los primeros no llegan a representar el 10\% del total, en ninguno de los años.

La distinción entre turistas nacionales y extranjeros sólo se pudo detectar en las dos últimas temporadas como consecuencia de la diferenciación en el precio de sus respectivas entradas. Esto reveló que - al menos en el período mencionado - los extranjeros representan menos del $40 \%$ del total de turistas.

Estas relaciones se establecieron sobre la cantidad efectiva de entradas vendidas, sin contemplar niños menores y otros exentos de pago, dado la dificultad de detectar su rango de distribución por origen.

Lo mismo ocurre en cuanto a la nacionalidad de los turistas extranjeros, cuya composición sólo se detecta a través de encuestas, las que, en general, revelan una alta incidencia de turistas europeos y norteamericanos, seguido por los provenientes de países limítrofes.

La distribución mensual de los turistas según nacionalidad indica que diciembre es el mes proporcionalmente más frecuentado por extranjeros, superando, en las dos temporadas, el 70\%. Le sigue en importancia el mes de noviembre, con algo más del $50 \%$, mientras que en los meses de septiembre y octubre hay un alto predominio porcentual del turista argentino (Tabla 2).
Tabla 2 Visitantes a la RNT Punta Tombo - Distribución según nacionalidad de los turistas

\begin{tabular}{|l|c|c|c|c|}
\hline \multirow{2}{*}{ Mes } & \multicolumn{2}{|c|}{ Temporada 1999/00 } & \multicolumn{2}{c|}{ Temporada 2000/01 } \\
\cline { 2 - 5 } & $\%$ arg. & \% extranj. & \% arg. & \% extranj. \\
\hline Septiembre & 84,34 & 15,66 & 86,26 & 13,74 \\
\hline Octubre & 77,65 & 22,35 & 80,10 & 19,90 \\
\hline Noviembre & 45,29 & 54,71 & 47,74 & 52,26 \\
\hline Diciembre & 26,30 & 73,70 & 29,13 & 70,87 \\
\hline Enero & 68,81 & 31,19 & 60,43 & 39,57 \\
\hline Febrero & 65,40 & 34,60 & 57,44 & 42,56 \\
\hline Marzo & 51,70 & 48,30 & 44,46 & 55,54 \\
\hline Abril & 53,60 & 46,40 & 67,29 & 32,71 \\
\hline Total & $\mathbf{6 3 , 5 6}$ & $\mathbf{3 6 , 4 4}$ & $\mathbf{6 1 , 5 2}$ & $\mathbf{3 8 , 4 8}$ \\
\hline
\end{tabular}


Sin embargo, analizado en términos absolutos, noviembre es el mes de mayor frecuencia en cuanto a la cantidad de turistas extranjeros $(+$ de 4 mil personas), mientras que octubre lo es para el turista argentino ( + de 9 mil personas) (Figura 4).

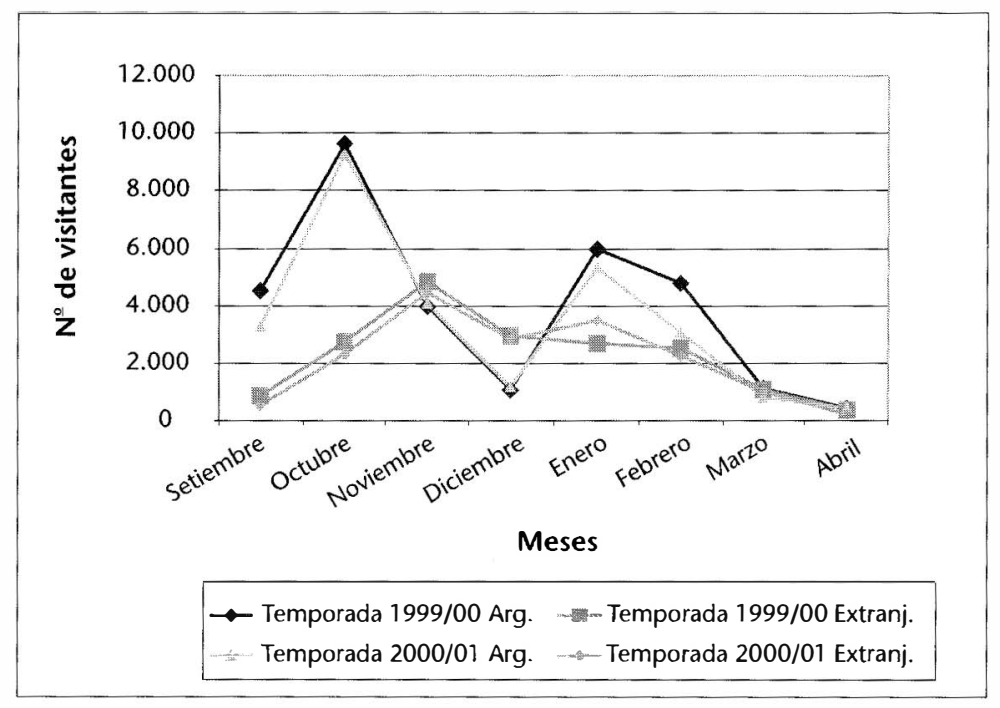

Figura 4 Visitantes a la RNT Punta Tombo - Turistas argentinos y extranjeros/ mes (Temporadas 1999/00 y 2000/01)

\section{Estacionalidad}

A los efectos de analizar el comportamiento estacional de la serie, se calculó el promedio mensual de visitas, considerando los datos suministrados por el Cuadro 1. Esto reveló una estacionalidad muy marcada, con la presencia de dos meses de demanda máxima (octubre y enero) seguidos, en importancia, por noviembre y febrero. (Figura 5).

Abriendo la totalidad de la serie, por mes y por año, se comprueba que se produjo un desplazamiento gradual en este tipo de comportamiento (Figura 6). En los últimos años, el mes de mayor presencia, en cuanto a la cantidad de turistas, fue octubre, en contraposición al mes de enero de los años precedentes.

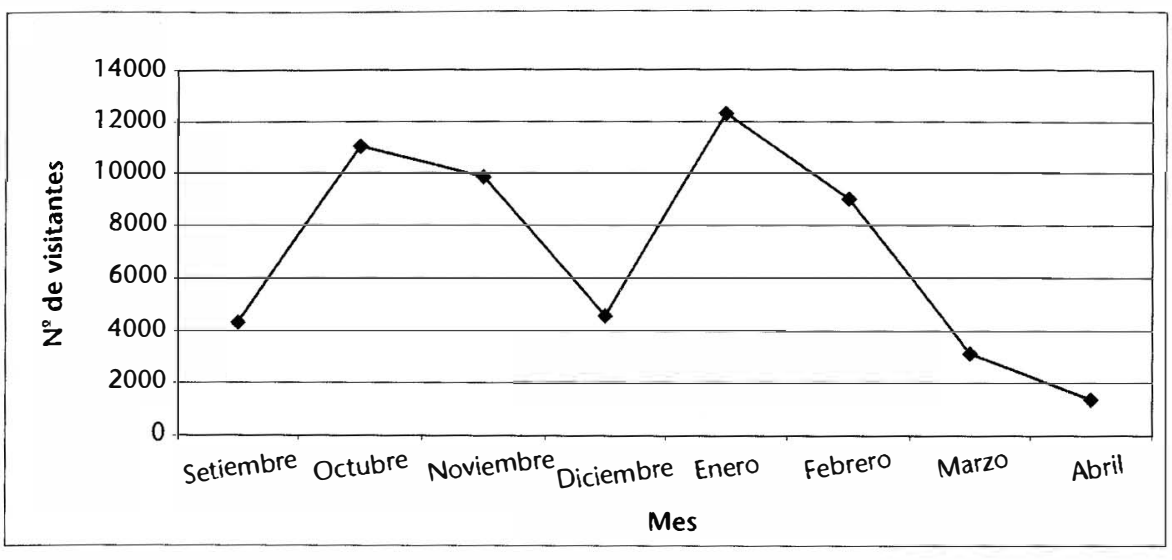

Figura 5 Visitantes a la RNT Punta Tombo - Promedia mensual de la serie

\section{Turistas / dias}

Para la temporada 1999/2000 se realizó un análisis del movimiento diario de visitantes, discriminados entre residentes, turistas argentinos y extranjeros (Figura 7).

Ese gráfico es de áreas acumuladas, o sea, que la línea superior representa la suma de los tres componentes mencionados. Éste permite observar las grandes fluctuaciones diarias en cuanto al número de visitantes. Resulta particularmente significativo el registro correspondiente al fin de semana largo del 12 de octubre, con 1.200 ingresantes a la Reserva (día 10/10), cifra que representa el doble del mayor ingreso alternativo de la temporada. Estos datos están basados sólo en la cantidad de ticket efectivamente vendida, por lo que la carga turística es aún mayor a lo que se observa en el gráfico.

En ese gráfico se corrobora lo expresado en el punto 3.2. acerca de la composición relativa de los diferentes subgrupos de visitantes según origen. Allí se aprecia que la mayor concentración de turistas extranjeros se produce, aproximadamente, en el lapso que media entre el 20 de octubre y el 20 de diciembre.

La carga diaria promedio de visitantes, para toda la temporada 1999/00, fue 221 personas. Pero, analizado mensualmente, es el mes de octubre donde se manifestó la máxima presión sobre el recurso, con 406 visitantes diarios. Le siguió enero con 308. 


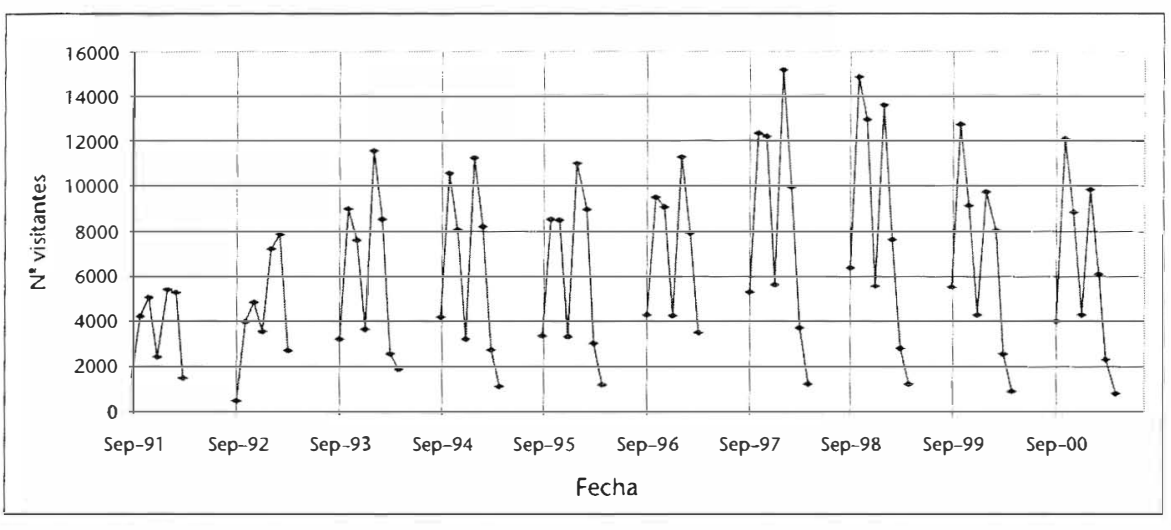

Figura 6 RNT Punta Tombo - Total de visitantes/mes (Período 1991-2001)

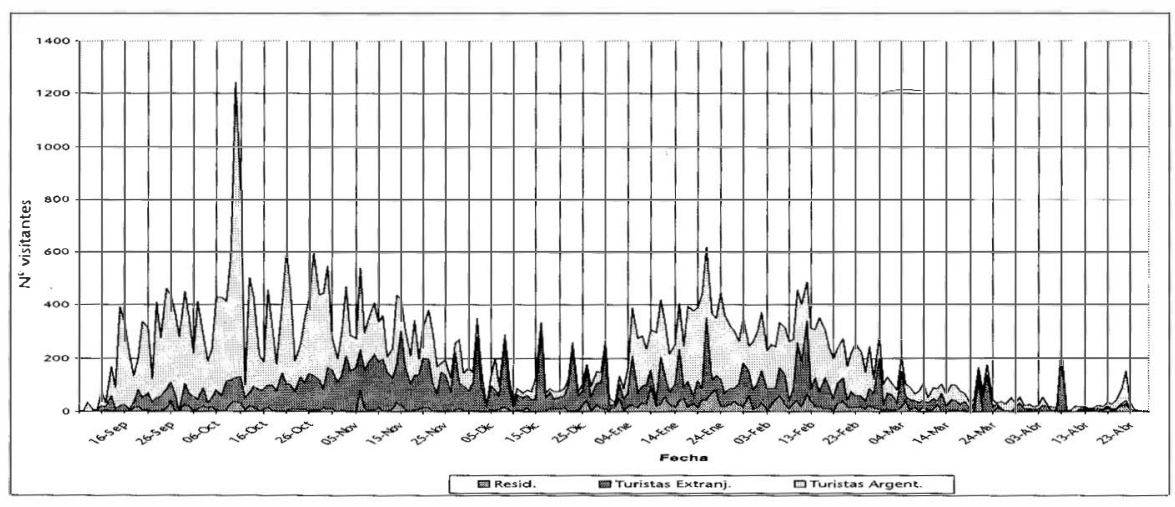

Figura 7 Visitantes a la RNT Punta Tombo - Número de visitantes y diarios y comparación con arribo de cruceros turísticos (Tempo-rada 1999/90)

\section{Influencia de los cruceros turísticos}

Los datos mencionados en el punto precedente se compararon con los días de arribo de cruceros turísticos a la ciudad de Puerto Madryn, cuya principal excursión en tierra consiste en visitar esta Reserva, señalados en el Gráfico 7 con una pequeña marca.

La llegada de turistas por este medio también evolucionó rápidamente en los últimos años. Considerando el mismo período que en el presente análisis, la tasa anual acumulada de crecimiento fue del 24,7\%. Así, partiendo de la cifra de 3.753 pax para la temporada 1991/92, se llegó a 27.402 pax en el 2000/01. En cuanto a la cantidad de embarcaciones, éstas pasaron de 13 a 34 en el mismo período.

El amarre de los cruceros se realiza en una época muy específica del año, que se extiende entre los meses de noviembre y marzo, observándose su mayor concentración (en número de pasajeros y en cantidad de barcos) entre los meses de enero y febrero.

Su estadía en puerto, por razones operativas y de capacidad del muelle, es muy breve -unas ocho horas, entre las $9 \mathrm{~h}$ y $17 \mathrm{~h}$ (Patagonia net, 2001:39) - por lo que el turista que decide hacer la excursión a Punta Tombo se encuentra sumamente limitado en tiempo, en razón de la distancia que debe recorrer (aproximadamente 180 kilómetros desde Puerto Madryn).

No existen registros fehacientes acerca de qué proporción de estos pasajeros realiza la mencionada excursión. Sin embargo, contando el total de turistas ingresantes a la Reserva en las fechas coincidentes al amarre de los cruceros turísticos - durante la temporada 1999/00 -, el mismo constituye sólo el $41 \%$ de la potencialidad de esta demanda.

\section{Ingresos monetarios directos}

A lo largo del período analizado, el valor del ticket de ingreso a la Reserva ha sufrido varias modificaciones, partiendo de un valor único de US $\$ 0,70$ para la temporada 1991/92. Actualmente, este precio es de US $\$ 2,00$ para los residentes y US $\$ 7,00$ para los turistas nacionales y extranjeros. Este incremento se ha dado en variasetapasy permitió un crecimiento constante y sostenido en el monto recaudado por este concepto, aún cuando se redujo el número de visitante al área. Sólo en la última temporada se produjo un descenso del $8 \%$ en la recaudación frente a una disminución en el $15 \%$ en la cantidad de visitantes (Tabla 3 y Figura 8.)

El crecimiento anual acumulado durante el período fue del $37,7 \%$, pero el mayor impacto se produjo en la temporada $92 / 93$, con un incremento del $416 \%$ sobre el registro de la temporada anterior. Esto fue consecuencia del cambio de un valor uniforme de $\$ 0,70$ a valores discriminados según diferentes segmentos de visitantes, a partir de enero de 1993. La tarifa más baja fue de $\$ 1,00$ (ó US\$ 1,00 ) para los residentes de la Provincia, $\$ 3,00$ para los jubilados y estudiantes y $\$ 5,00$ para el resto de los turistas nacionales y extranjeros. Los menores de 12 años estaban exentos de pago.

A partir de la temporada 1999/2000 estos valores se duplicaron, y se cambió la forma de segmentar a la población visitante. Los valores de ingreso a la Reserva para los residentes pasaron a $\$ 2,00$, turistas argentinos $\$ 5,00$ y turistas extranjeros 
Tabla 3 Evolución de los ingresos monetarios por venta de entrada a la Reserva

\begin{tabular}{|c|c|c|}
\hline Temporada & Ingresos (en miles \$) & Evolución \% \\
\hline $1991 / 92$ & 17,71 & \\
\hline $1992 / 93$ & 91,28 & 415,6 \\
\hline $1993 / 94$ & 191,12 & 109,4 \\
\hline $1994 / 95$ & 193,72 & 1,4 \\
\hline $1995 / 96$ & 196,06 & 1,2 \\
\hline $1996 / 97$ & 204,32 & 4,2 \\
\hline $1997 / 98$ & 265,18 & 29,8 \\
\hline $1998 / 99$ & 272,23 & 2,7 \\
\hline $1999 / 00$ & 345,15 & 26,8 \\
\hline $2000 / 01$ & 314,45 & $-8,9$ \\
\hline
\end{tabular}

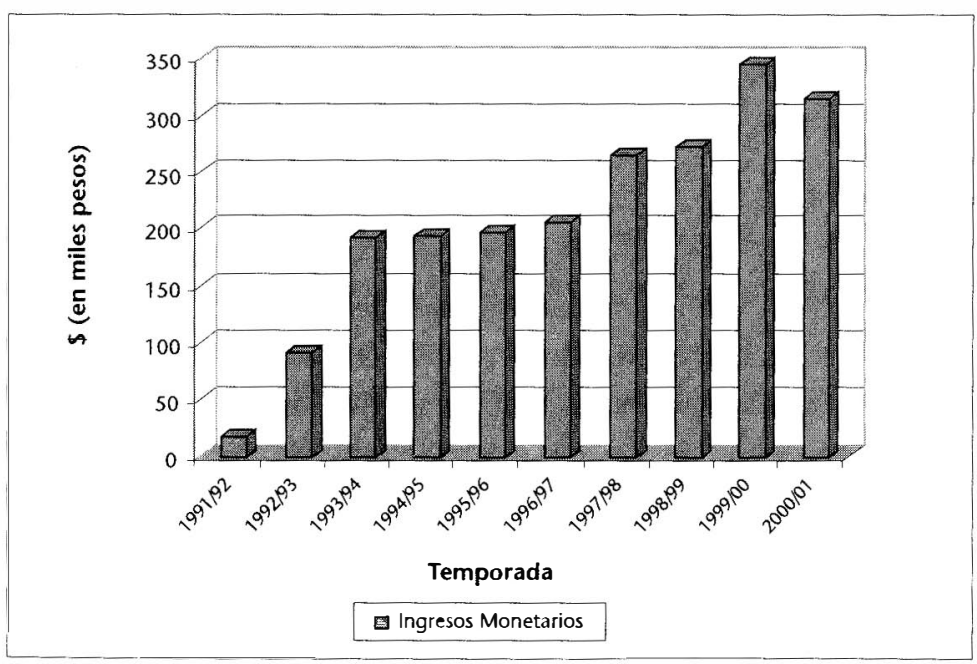

Figura 8 RNT Punta Tombo - Ingresos monetarios generados por venta de entradas a la Reserva
$\$ 10,00$. Pero, en enero de 2001 , hubo un nuevo giro en la política tarifaria, uniformándose el precio de los turistas en $\$ 7,00$ y reduciendo la edad de los niños exentos de pago a menores de 5 años.

Dado que los turistas argentinos son más numerosos que los extranjeros, este aumento del $40 \%$ en el valor de sus entradas permitió compensar en algo la caída de recaudación debido tanto al menor número de visitantes como a la reducción del $30 \%$ en el precio del ticket a extranjeros.

\section{Discusión y Conclusión}

A la luz de los resultados descriptos en el punto precedente, se observa la presencia de ciertos aspectos que merecen ser objeto de una mayor consideración y análisis en futuros estudios que se realicen sobre el área. Entre ellos se destacan:

- Ausencia de un criterio sistemático en cuanto a la aplicación de la política tarifaria referida al valor del ticket de ingreso al área protegida, lo cual redunda, en este caso particular, en la pérdida de continuidad de la fuente de información. Esto impide, a su vez, la realización de análisis de largo plazo y la aplicación de una clara segmentación del mercado;

- Aún es prematuro arriesgar una conclusión acerca del comportamiento decreciente de la demanda. No existen elementos suficientes para juzgar si éste se consolidará en el tiempo o no. Lo que sí se puede afirmar que este fenómeno afectó también al resto de los atractivos complementarios de la región. Es decir, los registros de ingresos de visitantes a la Reserva Natural Península Valdés mostraron cifras decrecientes durante los años 1999 y 2000 en tanto que los de cantidad de personas que realizaron el avistaje embarcado de ballenas también lo hicieron en el último año. Esto conduce a suponer que, en general, existe una menor demanda hacia los atractivos provinciales que pueden estar justificados, por un lado, en la profunda recesión económica que atraviesa Argentina y, por otro lado, puede haber razones inherentes a la promoción y comercialización del producto, debido a que esta reducción incluyó también los viajes de extranjeros al área;

- Sibien la comparación de la distribución mensual entre turistas argentinos y extranjeros sólo se realizó para dos temporadas, se observó una alta regularidad en el comportamiento de ambos grupos, de lo que se deduce que esa es la conducta habitual en los desplazamientos turísticospor la región; 
- El gradual cambio de máxima estacionalidad de la demanda dirigida a la Reserva Natural Turística de Punta Tombo del mes de enero al mes de octubre, puede tener explicación en el rápido crecimiento en la cantidad de turistas que realizaron el avistaje embarcado de ballenas durante la década de los '90, y que tiene en dicho mes su mayor expresión;

- La concentración diaria de visitantes en el mes de octubre - superior a la de cualquier otro mes - lleva a pensar en la capacidad de carga sobre el recurso y a la fragilidad biológica del mismo en función del momento crítico en cuanto a sus funciones reproductivas. Al respecto vale la pena citar el siguiente párrafo:

A la reserva ingresan otros pingüinos que no son reproductores porque son de madurez diferida. Son individuos jóvenes que llegan a partir de octubre o noviembre. En esta época hay un ingreso de pingüinos jóvenes que se debe tener en cuenta al momento de las visitas turísticas, dado que reaccionan de forma más sensible a la gente y no tienen ningún tipo de ataduras ni temores. Lo que hacen es inhibirse rápidamente, generando una gran perturbación. Corren y esto provoca que se asuste a pingüinos que están reproduciendo porque ven pasar la bandada, y se suman a la misma. El disturbio en esta época es más grande que al principio de temporada de incubación. (Yorio apud Albarracín y Arias, 2000);

- A través del análisis de la información, se ha demostrado que sólo concurre a la Reserva una porción inferior al $40 \%$ de los turistas que arriban a la costa del Chubut en cruceros turísticos. Esto indica que resultaría relativamente sencillo acrecentar la demanda de este segmento de visitantes, los que, por otra parte, ya están en territorio provincial;

- Existe una idea generalizada de que los turistas que arriban en cruceros comprometen seriamente la capacidad de carga de la Reserva provocando una concentración excesivamente alta de visitantes en días aislados. No obstante, se demostró que éste no es el único factor causante de tal circunstancia;

- Estos datos estarían indicando la necesidad de establecer un manejo más racional de la Reserva, regulando los horarios de ingresos y la cantidad horaria/diaria máxima de aceptación de visitantes, con un régimen especialmente acordado para los pasajeros de los barcos turísticos;

- En cuanto a la recaudación de divisas por venta de entradas a la Reserva, también se ha demostrado que el Estado ha sido particularmente eficaz en este aspecto, logrando acrecentar notablemente sus ingresos. Sería necesario demostrar, en este sentido, si se actuó con la misma eficacia reinvirtiendo estos ingresos en el mejoramiento de las instalaciones, en la atención a los visitantes y en estudios de impactos sobre el recurso a fin de asegurar el manejo sustentable del mismo.

A través del presente trabajo, se demostró cómo unos simples datos obtenidos de la venta de entradas para el ingreso a un área protegida pueden llegar a suministrar una cantidad de información cuya lectura revela tendencias y comportamientos sumamente específicos y vitalmente significativos a la hora de tomar decisiones de manejo, tanto en lo que se refiere a los recursos bióticos y abióticos del área como a las decisiones de inversión y de evaluación económica de los mismos.

\section{Referencias Bibliográficas}

ALBARRACÍN, I.; ARIAS, C. 2000. Aspectos ecológicos y biológicos de la RNPT. Informe técnico para el proyecto de investigación: Gestión ambiental de áreas protegidas: desarrollo de un modelo de gerenciamiento ambiental para la Reserva Natural Punta Tombo, Provincia de Chubut, República Argentina. Universidad Nacional de la Patagonia San Juan Bosco.

BOERSMA, Dee et al. 1990. Punta Tombo: recomendaciones de manejo. Nueva York: Wildlife Conservation International.

LOSANO, Piedad. 1986. Incidencia económica de las prácticas conservacionistas en la provincia del Chubut. Dirección General de Turismo y Recreación. Rawson.

PASCOTTO, M.; TAGLIORETTE, A.; LOSANO, Piedad. 1990. Península Valdés: lineamientos básicos plan de manejo. Organismo Provincial de Turismo. Rawson.

PATAGONIA NET. 2001. Revista de Negocios, Puerto Madryn, jun.

PROVINCIA DELCHUBUT, Dirección Provincial de Turismo. 1980. Plan de Manejo. Sistema Provincial de Conservación del Patrimonio Turístico. Tomo II. Rawson.

SARANDÓN, R. et. al. 2000. Fundamentos para el gerenciamiento ambiental de la Reserva Natural de Punta Tombo Chubut, $R$. Argentina. Documento de informe de avance del proyecto de investigación: Gestión ambiental de áreas protegidas: desarrollo de un modelo de gerenciamiento ambiental para la Reserva Natural Punta Tombo, Provincia de Chubut, República Argentina. Universidad Nacional de la Patagonia San Juan Bosco.

TAGLIORETTE, A.; LOSANO, P. 1996. Demanda turistica en áreas costeras protegidas de la Patagonia. Plan de Manejo Integrado de la Zona Costera Patagónica. Informe Técnico, Puerto Madryn: GEF/PNUD; WCS/FPN, $n^{2} 25$.

Recibido en 10/06/2003.

Aprobado en 25/09/2003. 